\title{
INNOVATION AND DIGITALIZATION IN TOURISM - RESTRICTION OR DEVELOPMENT FOR BUSINESS IN BULGARIA
}

\author{
E. Velikova* \\ Department „Economics of Tourism”, UNWE, Sofia, Bulgaria
}

\begin{abstract}
The purpose of this publication is to explore the opportunities that innovation and digitization provide in tourism. Although digitization is not a new direction in tourism, its understanding and use in a number of cases is hampered by the lack of knowledge and skills in the field. The aim has been achieved by exploring secondary sources on the specificities of innovation activity and digitization. Systematic approach, observation, abstraction, as well as some general science principles such as objectivity, dialectic, concretization, unity between theory and practice, system analysis, induction, deduction and others are also applied. A qualitative expert analysis of the websites of tourist sites has been carried out to determine the level of digitization in the tourism sector. The survey results show that tourist sites focusing on innovative supply to their customers use modern information technologies as well as technologies directly involved in improving the supply of tourism services, including traditional products, services and know-how. Consumer value is increased by providing product innovations, place and promotion. It comes to the conclusion that, despite the benefits of innovation and digitization, they are not a panacea that can solve all the emerging problems of today's business, and in many cases are a limiting factor.
\end{abstract}

Key words: tourist product, tourist service, users, trends, digital detox

\section{INTRODUCTION}

In recent decades, tourism has been one of the strongest and most rapidly developing and promising sectors of the economy. Data for recent years show an unprecedented increase in both travel, revenue, tourism and jobs. Bulgaria is no exception to the global trends. We are witnessing tourist demand of a new type that, by its scale, tendencies and essence, is the result of the growing demandingness of customers and the entry of new competitors with innovative models of work. The new market conditions provide development only to those destinations and even individual organizations that are flexible to the changing environment and able to anticipate the wishes of their customers.

Technologies and digitization, in turn, have changed our environment, our behavior and led to radical innovations in everyday life and the

*Correspondence to: Elenita Velikova, Department ,, Economics of Tourism”, UNWE, Sofia, Bulgaria, Students' City, "8 Dekemvri” Blvd, office 2136, +35928195441, evelikova@unwe.bg working environment. Tourism is not far from modern trends. Data glasses, voice control or the use of service robots are becoming more and more relevant. Real-time digitized services or unique design solutions provide special technical innovations and enhanced comfort. Undoubtedly the most favored by digital innovations is the luxury segment. In mid-class hotels, specialization on a given topic is crucial. In budget hotels, on the other hand, there is a concentration on individual services and everything that is not necessary has been abolished. The minimization of the services allows the entire stay to be controlled through a simple application that can be installed on the phone. Despite the many advantages of innovation and digitization, the penetration of technology creates both advantages and some difficulties that we will pay more attention to.

\section{PROBLEM SETTING}

Digitization in the service sector is becoming more and more important. It is not a new territory for tourism. Tourist products have long been provided through digital distribution systems. Our surveys show that a quarter of 
tourism businesses offer their products online and $50 \%$ of their revenue is generated through digital channels. (1)

Digitization offers many new opportunities that can be exploited by providers in the tourism industry. At the same time, competition is being intensified and companies have to keep pace with digitization in order to remain on the same level. The term of digitization is used in different interpretations: on the one hand, digitization is the "conversion of analog information in any form to digital form with suitable electronic devices so that the information can be processed, stored, and transmitted through digital circuits, equipment, and networks [2, cited in 3]." On the other hand, digitization is the process, which is caused by the adoption of digital technologies and the application systems that build upon them. Digitization can be defined according to different levels of intensity: from the pure presentation and information (website), the sales channel function (e-commerce), business process integration (E-Business) to new business models with virtual products or services $[4$, cited in 3$]$. (3)

Digitization is, to a great extent, a source of innovation in the field of tourism. Their research in the sphere of services is quite fragmented and has not received enough attention from the academic community to the present day. Innovation means using the knowledge to make business more profitable. The creation of a new platform, a new solution for the service provider, the creation of new services, a new value for the client, engage innovation in business partnerships, according to Andersen (2012) even stimulate managers to start a new business. (5)

Scientists also agree that customer engagement in the service process can refresh the service, create a new one, not only in the economy but also in the service industry. (6) This means thinking about the benefits of joint "production" of tourism services, which enables you to work, think, create and process together the customer and the supplier. The innovative radar developed by Sawhney et al. (2006) (7) can be considered a well-known and appropriate tool for analyzing service companies. This innovative radar contains four parts: a company's offering determines the customers it serves, the processes it uses, and the presence points.
The tourism industry is very heterogeneous and consists of many small and medium market players. It includes tour operators and tour guides, tourist destinations, but also service providers in the hospitality, transport, entertainment and congress sector. Moreover, digitization is no longer only applicable to placement. In recent years, the focus has been on communication. Professional websites and social networks accompany tourists during their travel. Innovations in digital products and processes are increasingly finding consumers in the tourism sector, for example as a digital concierge at the hotel reception or as a selfmonitoring document in the field of food hygiene.

New opportunities for tourism are so-called location-based services (eg route planning, weather and traffic information), and virtual reality creates different expectations for the service. But these applications bring with them new challenges. Questions are increasingly being asked: Who owns the data collected? Which data can be collected and stored at all? How can digital networks be created in destinations with benefits for tourists and tourist companies?

On the tourism market, players that are not connected with the sector are becoming more active: for example, appraisal and booking platforms that are gaining increasing market power. This influence is most felt in the hotel industry. Small private hotels are often dependent on large international distribution networks, and platforms such as AirBnB easily overcome existing regulations, creating preconditions for distortion of competition.

Digital applications are becoming more and more complex. Talking about the digital revolution and "Tourism 4.0". This means that the Internet, large data, artificial intelligence, robotics or 3D-pressure applications will not only change the industry but also take over the tourist services. Many small and medium-sized companies face major challenges. Particularly in rural areas there is a lack of the necessary infrastructure for the use of digital processes. Employee competence is often not sufficient, entrepreneurs' resources are limited, and investment funds are often not available to the extent necessary. Moreover, legal uncertainty (such as general conditions, data protection) makes businesses even more difficult. That is why tourism policy should determine the right 
VELIKOVA E.

conditions for successful development in the digital age.

Modern tourists are characterized by varying behaviors and characteristics. For this reason, targeting and segmentation of the market will continue to dominate the choice of marketing strategy on the part of tourism entrepreneurs. The market power of the hotelschains is steadily increasing, but steamhoteling offers the opportunity for more innovation, increasing the loyalty of the guests. People are increasingly emancipating as consumers and know exactly what they want and expect. They become "prosummers". Travelers increasingly prefer their own "IDestinations," which create new realities in tourism. It does not matter where tourists want to go, but what experiences they want to have. There is a revival of values. People are eager for proximity, for immediacy, for new simplicity. Demand for protection and security continues because of the increase in the number of unplanned disasters.

Security is becoming an essential theme for the hotel industry. Hotel chains offer high technical standards and the private hotel promises reliability. The gap between the poor and the rich continues to grow. The answer to the hotel industry is limited services, few services (reduction of basic services in the budget market) or full service, many services in the luxury hotel. The success of the brand and its denial are equally common. The recognition and denial of the mark proceed in parallel. The brand becomes more and more important because guests know it. At the same time, brands are no longer so interesting to the Internet generation and individualists.

All these changes in the tourism sector create requirements for tourism service providers. Tourism has the ability to quickly adapt the supply, depending on the changed market conditions. On the one hand, it offers highly digitized services to tourists looking for them. On the other hand, it can also offer an escape from a digitized environment in which we live and work anyway.

\section{RESULTS FROM THE STUDY}

Digital transformation is the most powerful trend of our time and affects all sectors of the economy. It affects almost all components of the value chain and can lead to rapid and sustained changes. "Industry 4.0" reveals significant opportunities for the manufacturing and service sectors, such as producing an 254 individual product or flexibly adapting the real-time value creation process.

To determine the level of digitization in the tourism sector, mainly secondary sources have been studied. Systematic approach, observation, abstraction, as well as some general science principles such as objectivity, dialectic, concretization, unity between theory and practice, system analysis, induction, deduction and others are also applied. A qualitative expert analysis of the websites of the tourist sites has been carried out, with the aim of establishing the level of digitization and innovation in the tourist companies in Bulgaria.

As a result of the surveys, we can summarize that digitization has a significant impact on the hospitality industry. It covers all business units and most parts of the value chain. It affects business models, communication structures, and business processes. Unfortunately, its application in Bulgaria is limited to the use of reservation and evaluation platforms, presence in social networks and collection of data for permanent clients. Multiple opportunities create enormous challenges for small and medium-sized hotels. On the one hand, they have to use common digital instruments, on the other hand, can provide only limited capacities of capital and staff. This circumstance requires an emphasis on the individual activities that are important to success. This includes a good breakfast in a pleasant atmosphere or friendly and helpful hotel staff.

Most businesses report enhanced web presence, which is a major advertising tool. As a result of the changed conditions, there is adaptability to new technical capabilities and consumer behavior. Websites must be creatively and technically programmed to meet the needs of mobile smartphone users. Many hotel websites do not meet this criterion. This, in turn, is a necessary condition for attracting the attention of potential users. The main question is what content should be included in these applications, and the modern virtual reality will further alter Web sites in a meaningful way. Good integration with additional service providers can increase business efficiency.

With the introduction of digital technologies, processes can be organized in a cost-effective and cost-efficient way. With their help you can save human resources and increase efficiency. Automation has a great application in terms of 
bookings and cancellations, which can minimize duplication and empty reservations. In addition, the processes of registration or distribution of personnel can be digitized. These processes are still at a very low level in tourist companies in Bulgaria.

In the "Future Hotel" project of Fraunhofer Institute $\operatorname{EAD}(8,9)$, complete projects for the development of hotel services were developed. The scenarios include fully automated placement and departure processes, adaptation of the room to the client's wishes (light, brightness, TV content, etc.) and network connection to the smartphone to manage certain functions (ordering breakfast, controlling air conditioning or work on the TV). Functions are controlled by different channels; especially suitable for mobile devices (smartphone, tablet, etc.) and smart TVs. Mobile devices may be provided to the customer or owned by them. The goal is to optimize services, increase the attractiveness of the hotel, and gather data for further business purposes.

In terms of bookings, an increasing number of customers use the internet. Nearly $50 \%$ of tourists plan their trip online today. More than $80 \%$ of all tourists have booked at least once a component (travel, accommodation, events, etc.) over the Internet. Digitization becomes more and more important during the stay. New forms of digital terrestrial communication offer advantages to both guests and hotel staff (9). The use of social networks is also increasing. Social media allow companies to advertise indirectly, for example, by posting and sharing photos or recommendations among Facebook users. Digital applications also offer the ability to actively advertise and expand distribution channels. Interesting is the fact that both the company itself and third parties can produce and share content. Accommodation providers should manage communication and advertising on these platforms and actively use the opportunities offered by social networks to promote their business. These include home page links, photos and videos as well as links to specific services, location description, and more.

However, this development also leads to dependence. For example, platforms (benchmarking, rating and rating portals) take the place between the customer and sometimes earn even more from the service provider. The booking fee via portals is often between 15 and
20 percent. This leads to the dominating position of reservation platforms. However, most small and medium-sized hotels rely heavily on platforms like Boeking to promote their product. Despite the desire to create their own web pages, Boeking is the most common and sometimes remains the only alternative for most accommodations in Bulgaria. Possible solutions are to offer additional benefits to customers if they book through the hotel's own site - such as a free breakfast, for example. Despite the risks and dependencies, the reservation and evaluation platforms will continue to dominate the tourism market in the future.

Customer-Relationship Management is increasingly digitizing in tourism. Customerspecific data is collected, analyzed and used for business purposes; on the one hand, and to offer interesting offers to hotel guests on the other. Data collected is usually for permanent customers.

Despite the many opportunities that digitalisation and innovation offer, Bulgarian tourism companies are still not well developed. Their experience is limited to the availability of their own web site, inclusion on some of the global booking platforms such as Booking.com or for evaluation like Tripadvisor, and the collection of information about the preferences and wishes of the regular customers.

Digitization has another side - people are becoming more and more overwhelmed and overwhelmed by its application everywhere. It is in this aspect that Bulgarian tourist companies have a greater chance of realization, namely escape from digitalisation.

One such niche is health. The pursuit of health will continue to grow in the coming years, and offering offers to meet health prevention or support will be a successful approach to developing and affirming businesses. The term "future health" in tourism therefore means to develop a new range of suggestions centered around "Selfness". This term means a major increase in life skills. Health is supported by four main areas: exercise (physical training), relaxation (wellness or regeneration), treatment (for prevention and symptomatology) and therapy. People want to learn to understand themselves and their bodies. This is the chance for hoteliers - to offer opportunities other than the worldwide digitization of the world. This also 
includes the possibility of affirming our small accommodation facilities.

\section{SOLUTIONS AND CONCLUSIONS}

Hotels have different opportunities to increase their innovative power. On the one hand, they can develop their product by modernizing rooms or flexible use of common areas by building them with removable walls. The hotel's new concepts use the lobby as a multifunctional room, in the evening as a bar and in the morning as a breakfast room. Higher investment in lobby equipment will be offset by more efficient recruitment. Also, technologies that offer strategic advantages, such as automated checking terminals or robots that can handle portions of room service, can also be used.

The potential of digitization is enormous and represents a great challenge for tourism companies. Through booking and rating portals, guests can get quicker, easier, and more fully informed about possible tourist destinations and accommodations. Therefore, companies have the task of constantly monitoring the current market developments, identifying new trends at an early stage and even more strongly thinking about the products and services offered from the client's point of view. But this process is also seen as an opportunity. Businesses should direct their potential target groups through an attractive website and popular social media (Facebook, Twitter, Instagram, etc.).

However, digitization should not be limited to the use of online channels. Rather than that, all processes in a company must be sequentially checked for the possibility of their digitization in order to exploit the opportunities they have obtained.

The so-called "Digital detox" is the real contrast to the growing tendency towards digitization. This is the theme of the world's largest ITB Fair in 2015. The daily life we live in will increasingly drive us to hotels that will offer us the opportunity to escape from reality and the continuous flood of information. This is a good opportunity for the development of the Bulgarian tourist companies, which in any case still do not use the possibilities of digitization. Which trend is going to be difficult to judge. At present, it is clear that those who are not involved in digitization will hardly be able to survive on the market in the long run.
The trends that will dominate the tourist supply in the future will be of interest. The Austrian SMG Trendforshung Tourism Research Institute (10) identifies six trends for the future of the hotel industry as a result of digitization.

Their observance is important for the success of the tourist sites in Bulgaria due to the still poor development of the sector in Bulgaria.

According to the first trend, "Tourists from tomorrow will not make a difference between their professional and personal lives. "This is related to increasing the creative approach in the work environment. Creative people constantly have ideas and can be inspired wherever they are. By 2020, more than 50\% of the working population will belong to the creative sector. This trend is linked to the need for hoteliers to decide whether the hotel will be the ideal work area or place to escape from everyday life - "online hotel" or "offline hotel". The next trend is related to the changed understanding of the luxury service, which is becoming more and more demanding. In the coming years, the average market segment, which generates nearly $50 \%$ of tourism turnover, will become stronger, although only $33 \%$ of travel is foreseen for it. By comparison, the low-cost segment generates $62 \%$ of travel, but only $26 \%$ of turnover. Luxury remains an interesting area for the tourism industry, as $27 \%$ of the turnover is due to $5 \%$ of tourists. But luxury will be defined in a new way. This segment looks for authenticity, declares quality of life as a primary goal, and focuses on individual services.

Interesting is the tendency for "Traveling to ourselves". For the guests of the future "I-the destination" is more than the place where they will spend their next vacation. Participation, insight, teamwork and experience are characteristic of her. Eco-hotels, for example, become more and more chic because tourists want to do something good for the environment during their vacation and at the same time do not give up anything. Learning has become a natural part of our lives, and sometimes we just have to travel to learn new things. Thus individual hotels can even become schools of the future. People are fond of real meetings and they can happen almost daily in hotels. The fourth dimension is the experience and more and more hotels are trying to offer it through the thematic. 
It does not matter that the hotels themselves are becoming destinations. With clever strategies and clear staging, the hotel is becoming a destination no matter where the world is. Concepts are offered that both provide privacy and high standards. Guests of the future are looking for rooms and rituals in which to feel comfortable. That is why the "IDestination" is perfectly complemented by hotels that promise exceptional events.

Our brain designs its memories through places. Places operate on three levels: the place and surrounding environment, architecture and events taking place in this place.

The fifth trend is connected to the network the journey begins on the net. The Internet is both positive and negative for the hotel industry: on the one hand, sobering comments, on the other hand recommendations and occasional bookings. Websites are mandatory for hotels, but they are not everything. They should provide access to the most important information and photos quickly and conveniently. Mobile applications (smartphones, tablets, etc.) can be used in a new way. Apps will also play an increasingly important role: entertainment and creativity are related to productivity.

According to the sixth trend, the future of the hotels is in the niche. Niche markets are future markets as more and more consumers are looking for differentiated experiences. It is important for the hotelier to assess which niche he can develop because of his employment. Nines must be experienced. In the last trend there is significant potential for hotels in Bulgaria. Offering a highly specialized product based on Bulgarian traditions and customs is a guarantee for success.

Summarizing and knowing the trends that will dominate the tourism market is a step towards the successful development of companies.

\section{CONCLUSION}

The boom in digitalisation began at the beginning of the millennium and will continue for the next few years. Social networks are increasingly taking the function of forming opinions. Tourism industry is also changing as a result of social networks. Hotel review sites have changed a lot. It is not so important for a hotel to be represented on all new social platforms but to take the new network seriously and have ready answers to attract customers. If we use technology to simplify client life, we have good chances for the future.

Apps are becoming more and more popular and there are more and more applications of hotels and tour operators. Hotel applications should be easy to use and, above all, useful: language guidance, wake-up calls, restaurant reservations, etc. Digitality must be everywhere and freely accessible. Offering a smart phone from the hotel with preinstalled applications, a small language and tourist guide and an integrated navigation system is a prerequisite for a good time.

But there are more and more niches that work beyond digital streams. Tourists are increasingly looking for the experience. They are aware of the real digital life that offers new connections and opportunities for multi-level entertainment, connects us with people in new ways and facilitates life through automation. The growing influence of digital media and network culture ultimately gives people a new freedom but also a greater responsibility in dealing with data and information.

The potential of digitization is enormous and presents a great challenge to tourism. At the time of booking and rating portals, tourists can get quicker, easier, and more fully informed about possible tourist destinations and accommodations. Therefore, companies have the task of constantly monitoring current market developments, identifying new trends at an early stage, and even more strongly thinking about offering products and services from the client's point of view. But this process is also seen as an opportunity.

Companies must target their potential target groups through an attractive website and popular social media (Facebook, Twitter, Instagram, etc.). An emerging trend for the next decade, for example, is the analysis of previously unused information (Big Data) in order to offer the perfect customer service. Innovation can also start with processes if, for example, information from customers' requests (breakfast requests, booking of services, etc.) is recorded and processed digitally for the development of new offers.

Companies need a focused online strategy. They must use the content, information and services of third parties (eg Tripadvisor) and integrate them on their websites. Equally 
important and useful is the fact that companies offer customers the opportunity to share their experience and to disseminate it online. In doing so, you must observe the entire journey of the users. However, digitization should not be limited to the use of online channels. Rather, all processes in a company must be scrutinized for the possibility of their digitization in order to make use of the resulting capabilities.

\section{REFERENCES}

1. Dredge, D., Phi, G., Mahadevan, R., Meehan, E. \& Popescu, E.S. (2018) Digitalisation in Tourism: In-depth analysis of challenges and opportunities. Low Value procedure GRO-SME-17-C-091-A for Executive Agency for Small and Mediumsized Enterprises (EASME) Virtual Tourism Observatory. Aalborg University, Copenhagen.

2. Businessdictionary (2017). http://www.businessdictionary.com/definiti on/digitization.html.

3. Potentials of Digitization in the Tourism Industry - Empirical Results from German Experts. Available from: https://www.researchgate.net/publication/3 18134641_Potentials_of_Digitization_in_th e_Tourism_Industry_-

_Empirical_Results_from_German_Experts [accessed Jun 13 2019].

4. Bauer, L., Boksberger, P., Herget, J.: The virtual dimension in tourism: criteria catalogue for the assessment of eTourism applications. In: O'Connor, P., Höpken, W.,
VELIKOVA E.

Gretzel, U. (eds.) Information and Communication technologies in tourism: proceedings of the international conference in Innsbruck 2008, pp. 522-532. Springer, Wien (2008).

5. Andersen J.B. (2012), Global spreading of knowledge and new rising knowledge centres, Conference 'Innovations 2012: How knowledge becomes business, 15.03. 2012, Vilnius.

6. Magnusson P.R. (2003), Benefits of involving users in service innovation, European Journal of Innovation Management 6 (4), pp. 228-238.

7. Sawhney M., Wolcott R.C., Arroniz I. (2006), The 12 different ways for companies to innovate, MIT Sloan Management Review 47 (3), pp. 74-81.

8. Fraunhofer-Institut für Arbeitswirtschaft und Organisation IAO (2009) FutureHotel Basics - Grundlagenwissen zur Hotellerie in Deutschland. Stuttgart, 2009.

9. Fraunhofer-Institut für Arbeitswirtschaft und Organisation IAO (2014) FutureHotel Small Medium: Situation und Perspektive der Individualhotellerie des 1-3 Sterne Segments im deutschsprachigen Raum Impulse und Handlungsempfehlungen für die Praxis. Stuttgart, 2014.

10.SMG Trendforschung. Hotel der Zukunft Die wichtigsten Trendfelder für die Hotellerie, Available from: https://www.hotelimpulse.at/asset/419270/ [accessed Jun 13 2019] 